\title{
Analysis of Machine Learning through Support Vector Machine: Catalyst
}

\author{
Pooja Shrimali \\ Department of computer science, RMTT College, \\ Udaipur, Rajasthan, India
}

\author{
K. Venugopalan \\ Department of Informatics and Computational \\ Sciences, \\ Mohanlal Sukhadia University, \\ Udaipur, Rajasthan, India
}

\begin{abstract}
This paper investigates the use of support vector machine (SVM) in machine learning. The purpose of this study is to experiment of SVM in e-learning methodology. Main constituent of this research is to innovate and implement pedagogical hypermedia document. In the article [19] artificial neural network (ANN) has been used to test learners learning capabilities, which is now being replaced by SVM in the present article to understand statistical analysis of learner's knowledge level. By this experiment it is suggested that this methodology is over and above ANN which is used as mathematical and statistical results.
\end{abstract}

\section{Keywords}

Machine learning, SVM, adaptive e-learning, learning objects

\section{INTRODUCTION}

The field of machine learning ( ML) is concerned with computer program that involuntarily develops to achieve appropriate knowledge [25]. Machine learning system is train and test by making use of a sample set of records. As the system has learned from the training data, it will be able to perform the essential function based on the learning curve. Generally, performance can be enhanced by additional training. Many literatures are offered on machine learning beneath their own individual vicinity of applications, owing to the diverse applications of machine learning.

Machine learning algorithms for extracting information may be divided widely into 2-categories:

Based on rational rules learning on one hand and second one is statistical learning, for each entity class, rule-based methods include a set of rules through training, at the other hand statistical system learn statistical models [26]. Systems based on rule or rational learning are BWI [7], (LP)2 [5], RAPIER [3], SNoW [20], SRV [17] and WHISK [21]. Few examples of subsequent statistical systems are HMMs [9], Maximal Entropy [4], and SVM [13].

In this research paper, for each learner personalization is attained through an adaptive assortment of learning resources. Based on the knowledge level each learner specified domain knowledge concepts. The learners will be assigned a combination of learning objects (LO) that according to their learning requirements, which will be based on their perceptive level of each particular domain knowledge concepts [18]. As per learning preferences and the cognitive style of the learner [12], the selection of the material will be supported by a set of teaching rules.

ANN is also the most widely used machine learning algorithm [1]. It is encouraged by genetic learning systems even though it does not imitate it completely. Back Propagation (BP) algorithm is the most popular which was used in self referenced article for evaluate the learners knowledge-level. ANN seeks to integrate 'human-like intelligence' inside computer systems.

Currently, a new learning methodology was discovered for evaluate learners knowledge level. That is called support vector machine (SVM), which was introduced to execute better performance than ANN in several cases. Moreover, in comparison with ANN, SVM can be mathematically derived as well as simpler to analyze. SVM functions by mapping and guiding data for learning- chore into an advanced dimensional aspect using kernel functions. In this way it may find a maximal margin on hyper plane for separating the data. To create the solution on hyper plane involves using quadratic equations $(\mathrm{QE})$ which is statistically intensive. However, understated method has been proposed to avoid the QE and made SVM learning realistic for many contemporary dilemmas. It can also be used to learn target functions. SVM is a data classification technique that is usually based on training and testing. Each training set instance has a target value and many attributes. SVM aims to produce a model to predict data instances target value in testing sets given as attributes alone. SVM based classification is done by many researchers [4]. Distinct from ANN, it is extremely sound established theory which is based on statistical learning [2]. Both SVM and ANN algorithms can be formulated in terms of learning machines.

The layout of this paper is presented in two sections, firstly presenting self referenced article briefly and secondly- to evaluate user knowledge through SVM, so as the statistical analysis can be done.

\section{PREVIOUS ARTICLE REFERENCE}

"Learner model" contains the learner's private details to find the best possible path throughout the preferences, earlier knowledge and their score detail taken as a whole experiment. According to the rules of "pedagogical model" navigation area becomes accustomed to the appropriate learning objects for the learner. Their score detail is also accumulated in the learner model. "Session model" maintains the static questionnaire for all the learners and then renews the learner model. "Course model" shows the Web course content according to the learners' behavior.

In other words, each learner can see their adapted and customized Web pages based on their areas of interest or interactions such as searching, bookmarking or setting preferences. AWBES can also assist the navigation area for every learner by adaptive system. 
Fig: 3 [self:[19] ] gives a synopsis over the system mechanisms and their communications. When the learner login to the AWBES, it links the session manager that launches the questionnaire. The information via questionnaire is used to initialize and create a learner model. When the learner has preferred the learning goal, the session manager sends this request to the course frame work. And then, system is accountable for deciding and assembling the content, which is proposed to learn. The course model links the domain ontology, in order to identify learning objects necessary for accepting the goal. Subsequently, it verifies the learner model in order to check previous knowledge. In the reference article, pedagogical model and ANN is used to first-rate and assemble the content in a method that is appropriate for the learner. The procedures are analyzed by evaluators that gauges and updates the learner model. When the learner logs out, then newer learner model is accumulated. The system is put into operation exclusively in ASP.NET and XML as a database. The neural network commotion is done under Matlab tool box.

In this article, SVM is being used in place of ANN. Through SVM one can estimate learner's knowledge level according to SVM parameters like sensitivity, specificity and support vectors. This is represented in a graphical model as under (Fig:1):--

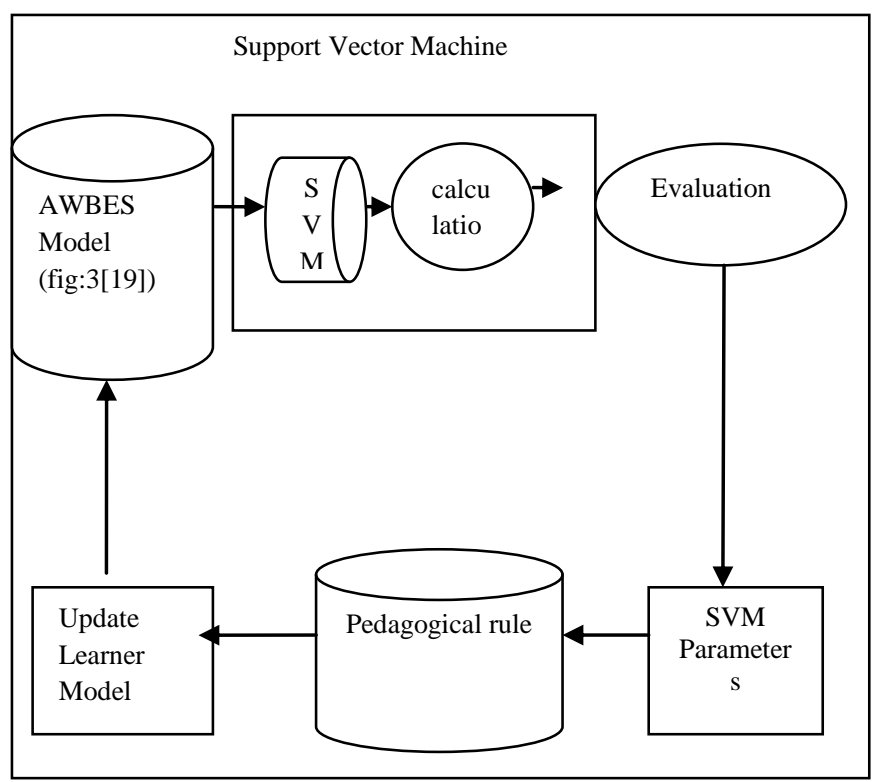

\section{SUPPORT VECTOR MACHINE}

Machine Learning is a synonymous of Artificial Intelligence and it is related to the improvement of technique and process which facilitates the computer to become skilled. In uncomplicated conditions expansion of algorithms which makes possible the machine to learn and execute tasks and activities [24]. It can enhance statistics in many ways. Many techniques and methodologies were developed over a period of time for this tasks [1].SVM is an example of Supervised Learning in one of the many classification of ML.

Support Vector Machine (SVM) was originally introduced in 1992, launched by Vapnik, Boser and Guyon in COLT-92[2]. SVM belongs to a kind of comprehensive and generalized linear classifiers. They are a set of associated organized learning techniques used for categorization and regression [1]. Additionally, SVM is a categorization and regression prediction device that makes use of machine learning theory that takes full advantage of prognostic precision while involuntarily shunning off the over-fit information. The definition of SVM is a hypothesis of linear function in a broad dimensions learnt from optimization principles so that it can be implement and derived from a statistical and mathematical equations. It gives a structure for analyzing the learner's previous knowledge, forecasting and adaptive learning from available learning materials. It helps to select the hyper plane space, so that it can be worked out in the target space [29].

A linear classifier has the form

$f(x)=w^{T} x+b$

$\mathrm{W}$ is known as the weight vector and $\mathrm{b}$ as the bias.

Binary Classification:-

Given training data $\left(\mathrm{x}_{\mathrm{i}}, \mathrm{y}_{\mathrm{i}}\right)$ for $\mathrm{i}=1 \ldots \mathrm{N}$, with $\mathrm{x}_{\mathrm{i}} \in \mathrm{R}^{\mathrm{d}}$ and $\mathrm{y}_{\mathrm{i}} \in\{-1,1\}$, learn a classifier $\mathrm{f}(\mathrm{x})$

such that

$$
\begin{aligned}
& \text { such that } \\
& \text { i.e. } \mathrm{y}_{\mathrm{i}} \mathrm{f}\left(\mathrm{x}_{\mathrm{i}}\right)>0 \text { for a eqrrect classification. }
\end{aligned}
$$

Thus, to get a function $\mathrm{f}(\mathrm{x})=\mathrm{y}: \mathrm{R}^{\mathrm{n}} \rightarrow\{+1,-1\}$ barring the fact that it appropriately classifies the patterns in the training data (a comparatively easier job), it also appropriately classifies hidden patterns too [22]. That is known as generalization [10]. In the experimental stage, modules are fed as input to SVM classifier. SVM is a binary classifier that is used to classify the modules into two classes. For AWBES two classes have been defined i.e. class 1 for grant permission to next level and class 0 for staying the same level.

\section{EXPERIMENT IN SVM}

In a machine learning method, SVM is a one of the important system for data analyzing and pattern recognition [14]. In AWBES Course Organization and Implementation Section were taught into adaptive environment. After Implementation Section, the SVM is a fruitful machine learning technique for adaptation. The adaptation method normally used to training and testing the learning objects those are available from the illustrations [23]. Each module in the training set consist one target and two input values. The main object of SVM is to give a statistical vales for analyze learners knowledge level those are given from the training data set. The thrust area of machine learning is the data categorizations and to create a hyper plane between classes. To obtain the best result for categorization and mapping from the machine learning, generate a largest distance in between support vectors and from the hyper plane [11].

In AWBES each lesson is divided into several modules and each module is sub divided into two sections. Each section composed through several LO. Each module score used for build a simple SVM using Matlab as an input and the target table. Through the performance parameters AWBES is able to analyze learner's actions in each module. Performance parameter 'sensitivity' gives the values for correct classified positive samples, in the other way, 'specificity' gives for correctly classified negative samples. True Positive (TP) is when LO's which are less than threshold values are correctly classified. True Negative (TN) is when the LO's which are above threshold values are correctly classified. False Positive (FP) is when LO's which are above threshold values are classified as less than threshold values. False Negative (FN) is when LO's which are less than threshold value are classified as above threshold value. Sensitivity measures the ability of 
the method to identify those LO's which are less than threshold value. Specificity measures the ability of the method to identify those LO's which are above threshold values. During the validation of classifiers 'Classperf' provides an interface to keep track of the performance. A classifier performance object optionally updates by 'Classperfcreates' [14]. The performance properties listed in the following code will perform Classifier's performance $(\mathrm{CP})$ :

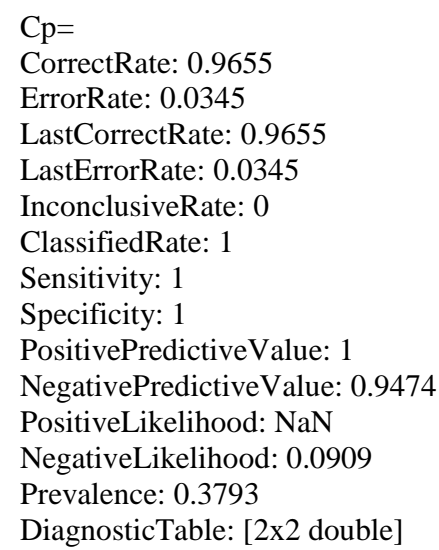

Code 1

Two kinds of samples are divided into SVM for training and testing through the following code fragment:-

[Train, Test $]=$ crossvalind $($ 'HoldOut', N, P) eq(iii)

For the evaluation of training and testing sets, results gives logical index vector for cross-validation of $\mathrm{N}$ [15].

In eq(iii) :-

$\mathrm{N}=$ No. of targets

$\mathrm{P}=0.5$ or $50 \%$ of target values
SVM classifier for the code fragment, structured information contains in the following system:-

svmStruct $=$ SupportVectors: $[14 \times 2$

double]

Alpha: [14x1 double]

Bias: -0.4718

KernelFunction: @linear_kernel

KernelFunctionArgs: \{\}

GroupNames: [31x1 double]

SupportVectorIndices:[14x1double]

ScaleData: [1x1 struct]

FigureHandles: $\{1 \times 3$ cell $\}$

\section{Code 2}

Code 1 and 2 shows the all classification performance parameters. Different scores of LO are classified by SVM with different kernel functions with different level of correct rate. The major strength of SVM is that the training of data is relatively easy. It balances reasonably well to elevated dimensional data and the trade-off amid classifier convolution and inaccuracy are controlled clearly [24].

From eq(i) ' $x$ ' is the support vector. Support vectors are the most difficult to classify data points that lie closest to the hyper plane and direct bearing on the optimum location of the hyper plane. From the svmtrain function it can be shown the optimal hyper plane. So that it can be train an SVM classifier by using a linear kernel function and plot the grouped data.

From the following code fragment the support vectors are plotted and then sorting is done using the test set as a support vector machine.

svmStruct=svmtrain(svmnewinpt(train,:),svmnewtargt(train),' showplot',true); eq(iv)

classes=svmclassify (svmStruct,svmnewinpt(test,:),'showplot',t rue); eq(v)

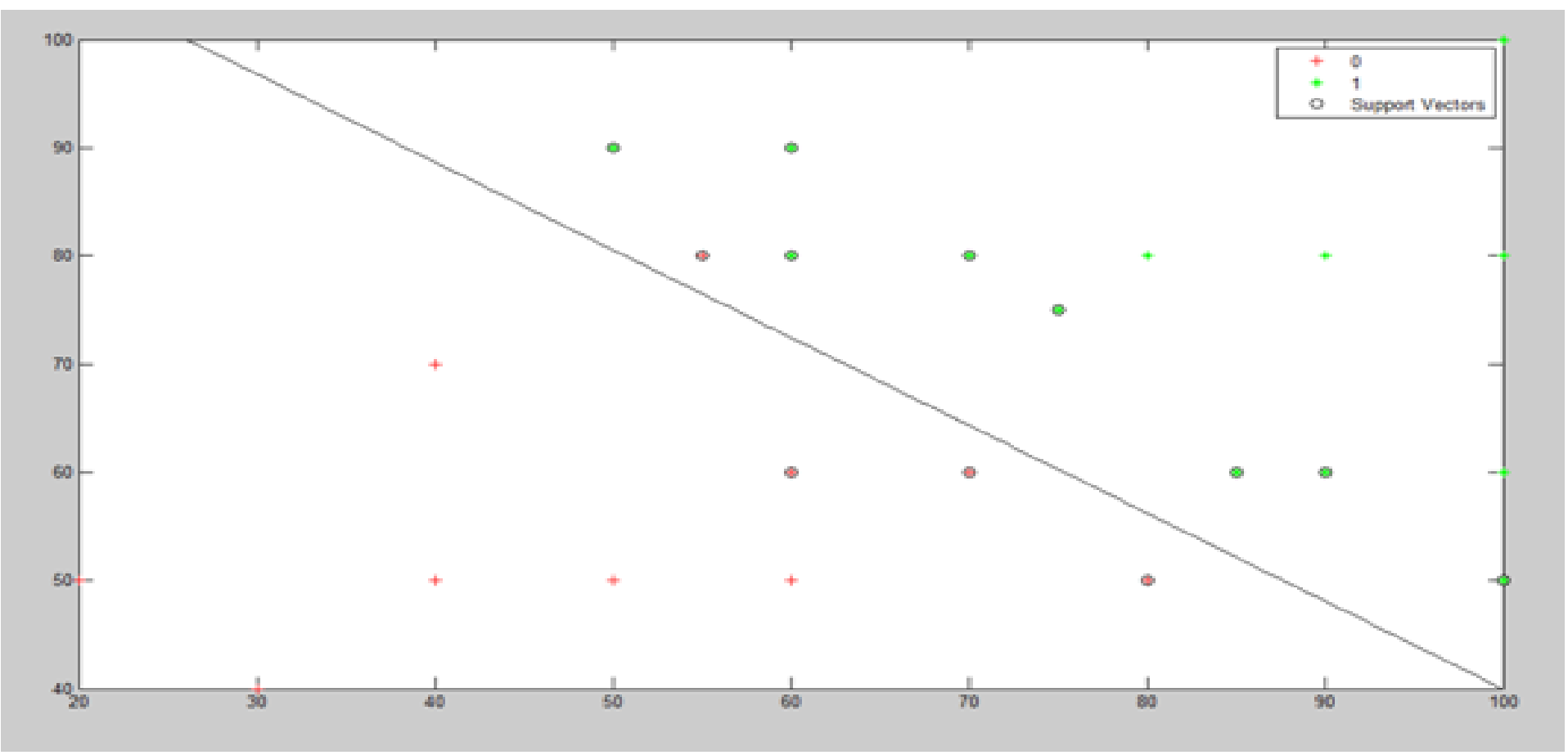

Fig:2: Support Vectors 


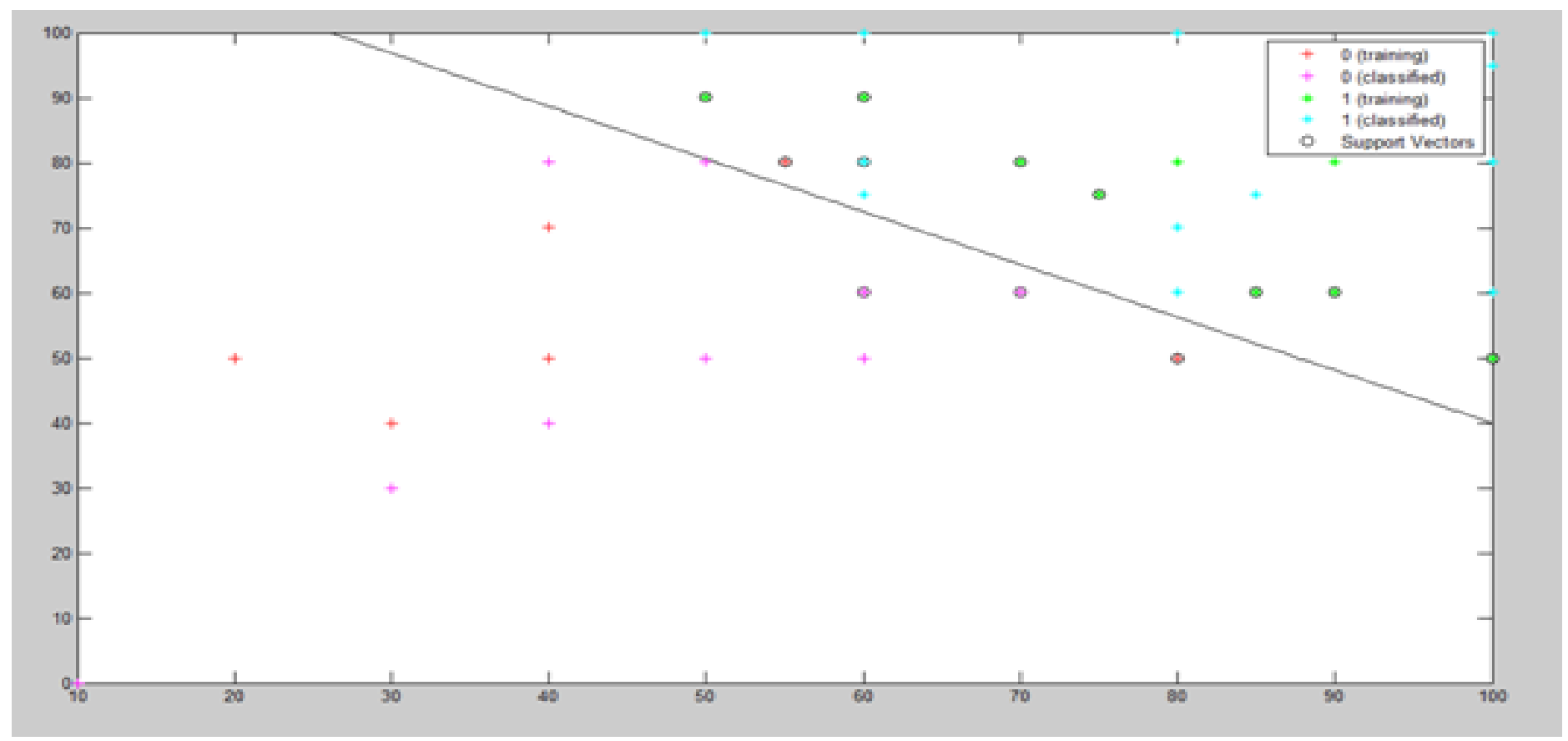

Fig 3: Classify test set using SVM

The entire methods are implemented in Matlab 7.6.0(R2008a) on window7. Computer configuration consists of Intel(R) Pentium(R) Dual CPU T2410@2.00GHz Processor, 2.00GB RAM 32 bit operating systems. The experiment data consists of 45 learner's test results. Experiment is conducted on each learner's module score which consists of two sections. Modules are the combination of the LO. According to the pedagogical model [19] there are three conditions 'Read again', 'Forward but Read Again' and 'Forward'.

Table 1: Pedagogical rules

\begin{tabular}{|l|l|l|l|}
\hline & Read again & $\begin{array}{l}\text { Forward but } \\
\text { read again } \\
\text { (fbr) }\end{array}$ & Forward \\
\hline LO's Score & $\begin{array}{l}\text { Less than } \\
60 \%\end{array}$ & $\begin{array}{l}\text { Between 60 } \\
\text { to 80\% }\end{array}$ & Above 80\% \\
\hline $\begin{array}{l}\text { Target read } \\
\text { again table }\end{array}$ & 1 & 0 & 0 \\
\hline $\begin{array}{l}\text { Target fbr } \\
\text { table }\end{array}$ & 0 & 1 & 0 \\
\hline $\begin{array}{l}\text { Forward } \\
\text { table }\end{array}$ & 0 & 0 & 1 \\
\hline
\end{tabular}

The LO's scores were partitioned arbitrarily into training set, testing set with equal numbers. Score of module's in form of score of LO's are passed as input to SVM and three times according to Table 1 each table is passed as target table then each table's classification performance is measured. Subsequently, three SVMStruct models are obtained and then they are compared with each SVMStruct models parameters. Using Sensitivity and Specificity the learner's knowledge level is analyzed. If the Sensitivity and Specificity obtained is 1 or near about 1 , then the learner is eligible for next module. In some cases if the values of specificity and sensitivity are same then the support vectors are also considered as the complexity is propositional to the total no of support vectors.

\section{CONCLUSION}

This paper presents the capability of the SVM to the evaluation of learner's knowledge level. In AWBES, the ANN is functioning to evaluate learner's knowledge level likewise SVM also contributes to assess the learner's capability by machine learning algorithm. The above methodology successfully adopted in selecting learning objects for a learner based on domain ontology with the perfection. Briefly it is proved that the SVM is mathematical and statistical analysis of adaptive learning to achieve perfect results. It is concluded here the SVM is full proved learning system in machine learning to assess more correct results.

\section{REFERENCES}

[1] Abdul Rahim Ahmad1 Marzuki Khalid2 Rubiyah Yusof2 "Machine Learning Using Support Vector Machines" 1Universiti Tenaga Nasional Km 7, Jalan KajangPuchong, $43009 \quad$ Kajang, $\quad$ Selangor. abdrahim@uniten.edu.my Centre for Artificial Intelligence and Robotics Universiti Teknologi Malaysia Jalan Semarak, 54100 Kuala Lumpur \{marzuki,rubiyah\}@utmkl.utm.my

[2] B. E. Boser, I. M. Guyon, and V. N. Vapnik "A Training Algorithm for Optimal Margin Classifiers." In D. Haussler, editor, $5^{\text {th }}$ Annual ACM Workshop on COLT, pages 144-152, Pittsburgh, PA, 1992. ACM Press.

[3] Cali_, M. E.” Relational learning techniques for natural language information extraction." $\mathrm{PhD}$ thesis, University of Texas at Austin, 1998

[4] Chieu, H. L., Ng, H. T."Named entity recognition: A maximum entropy approach using global information." In Proceedings of the $19^{\text {th }}$ International Conference on Computational Linguistics (COLING'02), Taipei, Taiwan, 2002

[5] Ciravegna, F.: (LP)2, "an adaptive algorithm for information extraction from web related texts." In 
Proceedings of the IJCAI-2001 Workshop on Adaptive Text Extraction and Mining, Seattle, 2001

[6] Duda R. and Hart P. "Pattern Classification and Scene Analysis", Wiley, New York 1973.

[7] Freitag, D.’Machine Learning for Information Extraction in Informal Domains." PhD thesis, Carnegie Mellon University, 1998.

[8] Freitag, D., Kushmerick, N." Boosted Wrapper Induction." In Proceedings of AAAI 2000, 2000.

[9] Freigtag, D., McCallum, A. K."Information extraction with HMMs and shrinkage." In Proceedings of Workshop on Machine Learning for Information Extraction, pages 31\{36, 1999

[10] Henok Girma "A Tutorial on Support Vector Machine" Center of expermental mechanichs University of Ljubljana 2009

[11] Kan Xie "Support Vector Machine Concept and matlab build"

[12] Karampiperis , P., and Sampson, D. (2005). “Adaptive Learning Resources Sequencing in Educational Hypermedia Systems." Educational Technology \& Society, 8(4), 128-147.

[13] Isozaki, H., Kazawa, H.”Efficient Support Vector Classifiers for Named Entity Recognition." In Proceedings of the 19th International Conference on Computational Linguistics (COLING'02), pages 390\{396, Taipei, Taiwan, 2002

[14] MathWorks “Classperf"

[15] MathWorks "Crossvalind"

[16] May eld, J., McNamee, P., Piatko, C."Named entity recognition using hundreds of thousands of features." InWalter Daelemans and Miles Osborne, editors, Proceedings of CoNLL-2003, pages 184\{187. Edmonton, Canada, 2003.
[17] Nello Cristianini and John Shawe-Taylor, "An Introduction to Support Vector Machines and Other Kernel-based Learning Methods", Cambridge University Press, 2000

[18] Norsham Idris1, Norazah Yusof2, Siti Zaiton Mohd. Hashim3 "A Model for Adaptive Selection of Learning Material in an Intelligent Learning System using combination of Supervised and Unsupervised Machine Learning Techniques" Faculty of Computing, Universiti Teknologi Malaysia, Skudai, Johor, MALAYSIA

[19] Pooja Shrimali, K. Venugopalan, Prabha Vajpeyee "Study and Analysis of an Adaptive Web Based ELearning System" IJCA Issue 4, Volume 3 (May - July 2014) www.rspublication.com/ijca/ijca_index.htm

[20] Roth, D., Yih, W. T."Relational learning via propositional algorithms: an information extraction case study." In Proceedings of the Seventeenth International Joint Conference on Arti_cial Intelligence (IJCAI), pages $1257\{1263,2001$.

[21] Soderland, S.’'Learning information extraction rules for semi-structured and free text. Machine Learning", 34 (1999) $233\{272$

[22] Steven Busuttil "Support Vector Machines" Department of Computer Science and AI,University of Malta

[23] Theodoros Evgenuiu and Massimilliano Pontil"Statistical Learning Theory",a Primer 1998.

[24] Vikramaditya Jakkula "Tutorial on Support Vector Machine (SVM)" Vikramaditya Jakkula, School of EECS, Washington State University, Pullman 99164.

[25] Wikipedia Online. Http://en.wikipedia.org/wiki

[26] Yaoyong Li, Kalina Bontcheva, Hamish Cunningham "SVM Based Learning System for Information Extraction." 